\title{
Spectroscopy of doubly and triply-charmed baryons from lattice QCD
}

\section{Padmanath*}

Tata Institute of Fundamental Research, Mumbai

E-mail: padmanathetheory.tifr.res.in

\section{Robert G. Edwards}

Jefferson Laboratory, VA

E-mail: edwards@jlab.org

\section{Nilmani Mathur}

Tata Institute of Fundamental Research, Mumbai

E-mail: nilmanidtheory.tifr.res.in

\section{Michael Peardon}

Trinity College, Dublin

E-mail: mjp@maths.tcd.ie

\begin{abstract}
We present the ground and excited state spectra of doubly and triply-charmed baryons by using lattice QCD with dynamical clover fermions. A large set of baryonic operators that respect the symmetries of the lattice and are obtained after subduction from their continuum analogues are utilized. Using novel computational techniques correlation functions of these operators are generated and the variational method is exploited to extract excited states. The lattice spectra that we obtain have baryonic states with well-defined total spins up to 7/2 and the low lying states remarkably resemble the expectations of quantum numbers from $\mathrm{SU}(6) \otimes \mathrm{O}(3)$ symmetry. Various energy splittings between the extracted states, including splittings due to hyperfine as well as spin-orbit coupling, are considered and those are also compared against similar energy splittings at other quark masses. Using those splittings for doubly-charmed baryons, and taking input of experimental $B_{c}$ meson mass, we predict the mass splittings of $B_{c}^{*}-B_{c}$ to be about $80 \pm 8 \mathrm{MeV}$ and $m_{\Omega_{c c b}}=8050 \pm 10 \mathrm{MeV}$.
\end{abstract}

$31^{\text {st }}$ International Symposium on Lattice Field Theory LATTICE 2013

July 29 - August 3, 2013

Mainz, Germany

\footnotetext{
*Speaker.
} 


\section{Introduction}

The recent discovery of numerous hadrons at various particle colliders, like Belle, BaBar, $\mathrm{CDF}, \mathrm{LHCb}, \mathrm{BECIII}$ etc., has brought a resurgence of interest in the heavy hadron spectroscopy [1]. However, in contrast to heavy quarkonia which have been studied comprehensively, heavy baryons have not been explored in much greater detail, both theoretically and experimentally, though the later can also provide similar information about the quark confinement mechanism as well as elucidating our knowledge about the nature of strong force by providing a clean probe of the interplay between the perturbative and the non-perturbative QCD. Experimentally only a handful of singly charmed baryons have been discovered [1]. Experimentally the discovery of doubly charm baryon is controversial [1], whereas no triply heavy baryon has been observed yet. Moreover most of the observed charmed baryons do not have assigned quantum numbers yet. However it is expected that the large statistical sample that will be collected in experiments at BES-III, the LHCb, and the planned PANDA experiment at GS/FAIR may provide significant information for baryons with heavy quarks. In light of these existing and future experimental prospects on charm baryon studies, it is desirable to have model independent predictions from first principles calculations, such as from lattice QCD. Results from such calculations can be compared with those obtained from potential models which have been very successful in the case of charmonia and will naturally provide crucial inputs to the future experimental discovery. Various lattice QCD calculations were performed to compute only the ground states of various charm hadrons with spin up to $\frac{3}{2}$, including quenched $[2,3]$ as well as full QCD [4-9]. Here we present a comprehensive study of the triply and doublycharmed baryon spectra with spin up to $\frac{7}{2}$ for both parities. In addition we also discuss the quark mass dependence of various energy splittings among them due to hyperfine interactions as well as for spin-orbit coupling. The details work on triply charmed spectra was reported in Ref. [10].

\section{Numerical details}

In recent years the Hadron Spectrum Collaboration (HSC) has exploited a dynamical anisotropic lattice formulation to extract highly excited hadron spectra. Adopting a large anisotropy coefficient $\xi=a_{s} / a_{t}=3.5$, with $a_{t} m_{c} \ll 1$, it ensures that the standard relativistic formulation of fermions can also be used for charm quarks. Along with Symmanzik-improved gauge action the $N_{f}=2+1$ flavours fermionic fields were described using an anisotropic Shekholeslami-Wohlert action with tree-level tadpole improvement and stout-smeared spatial links. The temporal lattice spacing, $a_{t}^{-1}=5.67 \mathrm{GeV}$, was determined by equating the $m_{\Omega}$ to its physical value, resulting in a lattice spatial extension of $1.9 \mathrm{fm}$, which should be sufficiently large for a study of triply-charmed baryons. More details of the formulation of actions as well as the techniques used to determine the anisotropy parameters can be found in Refs. [11, 12]. The lattice action parameters of the gauge field ensembles used in this work are given in Table 1.

\begin{tabular}{cccc|cc|cc}
\hline Lattice size & $a_{t} m_{\ell}$ & $a_{t} m_{s}$ & $N_{\text {cfgs }}$ & $m_{\pi} / \mathrm{MeV}$ & $a_{t} m_{\Omega}$ & $N_{\text {tsrcs }}$ & $N_{\text {vecs }}$ \\
\hline $16^{3} \times 128$ & -0.0840 & -0.0743 & 96 & 396 & $0.2951(22)$ & 4 & 64 \\
\hline
\end{tabular}

Table 1: Details of the gauge-field ensembles used. $N_{\text {cfgs }}$ is the number of gauge-field configurations while $N_{\text {tsrcs }}$ and $N_{\text {vecs }}$ are the number of time sources per configuration and the number of eigenvectors used for each time source in the distillation method, respectively. 


\begin{tabular}{c|cccccc}
\hline \multirow{2}{*}{} & \multicolumn{2}{|c}{$G_{1}$} & \multicolumn{2}{c}{$\mathrm{H}$} & \multicolumn{2}{c}{$G_{2}$} \\
\cline { 2 - 7 } & $g$ & $u$ & $g$ & $u$ & $g$ & $u$ \\
\hline$c c c$ & 20 & 20 & 33 & 33 & 12 & 12 \\
$c c c_{h}$ & 4 & 4 & 5 & 5 & 1 & 1 \\
$c c c_{n r}$ & 4 & 1 & 8 & 1 & 3 & 0 \\
\hline$c c q$ & 55 & 55 & 90 & 90 & 35 & 35 \\
$c c q_{h}$ & 12 & 12 & 16 & 16 & 4 & 4 \\
$c c q_{n r}$ & 11 & 3 & 19 & 4 & 8 & 1 \\
\hline
\end{tabular}

(a)
Non-Relativistic : $\mathrm{SU}(6) \otimes \mathrm{O}(3)$

\begin{tabular}{c|ccccc|cccc}
\hline \multicolumn{8}{|c|}{$c c c$} & \multicolumn{5}{c}{$c c q$} \\
\hline & $\mathrm{J}$ & $1 / 2$ & $3 / 2$ & $5 / 2$ & $7 / 2$ & $1 / 2$ & $3 / 2$ & $5 / 2$ & $7 / 2$ \\
\hline 0 & & 0 & 1 & 0 & 0 & 1 & 1 & 0 & 0 \\
\hline 1 & 1 & 1 & 0 & 0 & 3 & 3 & 1 & 0 \\
\hline $2 h$ & 1 & 1 & 0 & 0 & 3 & 3 & 1 & 0 \\
\hline 2 & 2 & 3 & 2 & 1 & 6 & 8 & 5 & 2 \\
\hline
\end{tabular}

(b)

Table 2: (a) Total number of operators used for $c c c$ and $c c q$ baryons in each lattice irrep. (b) The description of the non-relativistic operators used. D stands for the number of derivatives, while $\mathrm{J}$ represents the continuum spin. In both $h$ and $n r$ stands for the hybrid and non-relativistic operators respectively.

\subsection{Operator construction and spin identification}

Lattice computations of hadron masses proceed through the calculations of the Euclidean two point correlation functions, between creation operators at time $t_{i}$ and annihilation operators at time $t_{f}$.

$$
C_{i j}\left(t_{f}-t_{i}\right)=\left\langle 0\left|O_{j}\left(t_{f}\right) \bar{O}_{i}\left(t_{i}\right)\right| 0\right\rangle=\sum_{n} \frac{Z_{i}^{n *} Z_{j}^{n}}{2 m_{n}} e^{-m_{n}\left(t_{f}-t_{i}\right)}
$$

The RHS is the spectral decomposition of such two point functions where the sum is over a discrete set of states. $Z^{n}=\left\langle 0\left|O_{i}^{\dagger}\right| n\right\rangle$ is the vacuum state matrix element, also called as overlap factor. We use a large basis of operators, constructed employing derivative-based operator construction formalism [13], including non-local operators constructed using up to two derivatives by which we are able to realize states up to spin $J=7 / 2$ for both the parities. Further the quark fields in these operators were distilled so as to compute the matrix of correlation functions with reduced contamination from the UV modes in the low energy physics that we are interested in [14]. The continuum states get subduced [13] over the irreps of the symmetry of the lattice $\left(\mathscr{O}_{h}\right)$. For each of these irreps, we compute $N \times N$ matrix of correlation functions, where $\mathrm{N}$ is the number of operators used in each irrep as tabulated in the Table 2(a). Here $g$ and $u$ represents positive and negative parity. Table 2(b) gives the details of the subset of operators that are formed just by considering only the upper two components of the four component Dirac-spinors. This subset of operators are called nonrelativistic as they form the whole set of creation operators in a leading order velocity expansion.

We employ a variational method [15] to extract the spectrum of baryon states from the matrix of correlation functions calculated by using the large basis of interpolating operators discussed in the previous subsection. The method proceeds by solving a generalized eigenvalue problem of the form

$$
C_{i j}(t) v_{j}^{(n)}\left(t, t_{0}\right)=\lambda^{(n)}\left(t, t_{0}\right) C_{i j}\left(t_{0}\right) v_{j}^{(n)}\left(t, t_{0}\right)
$$

where the eigenvalues, $\lambda^{(n)}\left(t, t_{0}\right)$ form the principal correlators and the eigenvectors are related to the overlap factors as $Z_{i}^{(n)}=\left\langle 0\left|O_{i}\right| n\right\rangle=\sqrt{2 E_{n}} \exp ^{E_{n} t_{0} / 2} v_{j}^{(n) \dagger} C_{j i}\left(t_{0}\right)$. The energies are determined by fitting the principal correlators, while the spin identification of the states are made by using these overlap factors as discussed in ref.[15].

\section{Results}

In Figure 1(a) we show the spin identified spectra of the triply charmed baryons where $3 / 2$ times the mass of $\eta_{c}$ is subtracted to account for the difference in the charm quark content [10]. It is preferable to compare the energy splittings between the states, as it reduces the systematic uncertainty in the determination of the charm quark mass parameter in the lattice action and to 


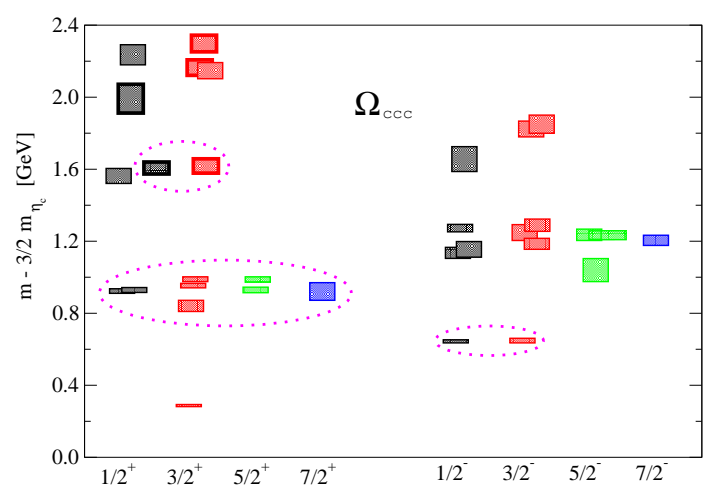

(a)

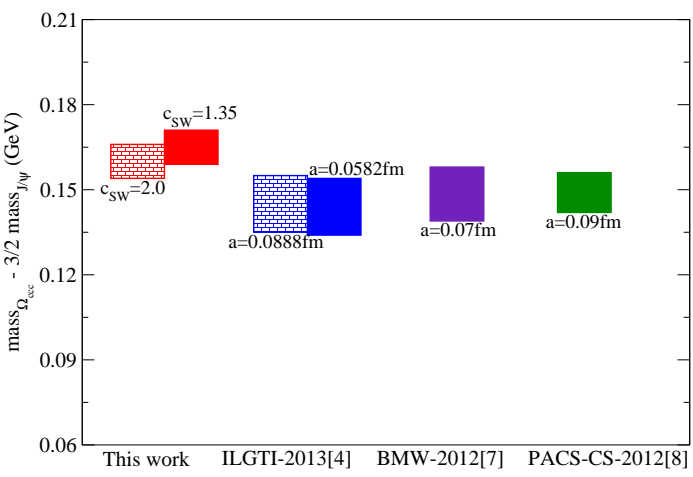

(b)

Figure 1: (a) Spin identified spectra of triply-charmed baryons with respect to $\frac{3}{2} m_{\eta_{c}}$ mass. The boxes with thick borders corresponds to the states with strong overlap with hybrid operators. The states inside the pink ellipses are those with relatively large overlap to non-relativistic operators. (b) Mass splitting of the ground state of $J^{P}=\frac{3}{2}^{+} \Omega_{c c c}$ from $\frac{3}{2}$ times the mass of $J / \psi$ meson is compared for various lattice calculations.

lessen the effect of ambiguity in the scale setting procedure. Boxes with thicker borders correspond to those with a greater overlap onto the operators that are proportional to the field strength tensor, which might consequently be hybrid states [16]. The states inside the pink ellipses have relatively large overlap with non-relativistic operators and should thus be well described in a quark model. One remarkable feature that one can observe is the number of states in the non-relativistic band exactly agree with expectations shown in Table 2(b). This agreement between the spectra and the expectations based on a model with the non-relativistic quark spins provides a clear signature of $\mathrm{SU}(6) \otimes \mathrm{O}(3)$ symmetry in the spectra.

To assess the effect of radiative corrections on the co-efficient of the improvement term in the charm quark action which could lead to significant change in the physical predictions, a second calculation was carried out after the co-efficient was boosted from the tree-level $c_{s}=1.35$ to $c_{s}=$ 2.0. As was seen for the $1^{--}$and other excited states in the charmonium study [17], we observe a shift in the energy difference, $m_{\Omega_{c c c}}-3 / 2 m_{\eta_{c}}$, to be approximately $45 \mathrm{MeV}$ [10]. In Figure 1(b) we compare our results for $m_{\Omega_{c c c}}-3 / 2 m_{J / \psi}$ with other lattice calculations which use different discretization and so have distinct artefacts. Our results are consistent other results.

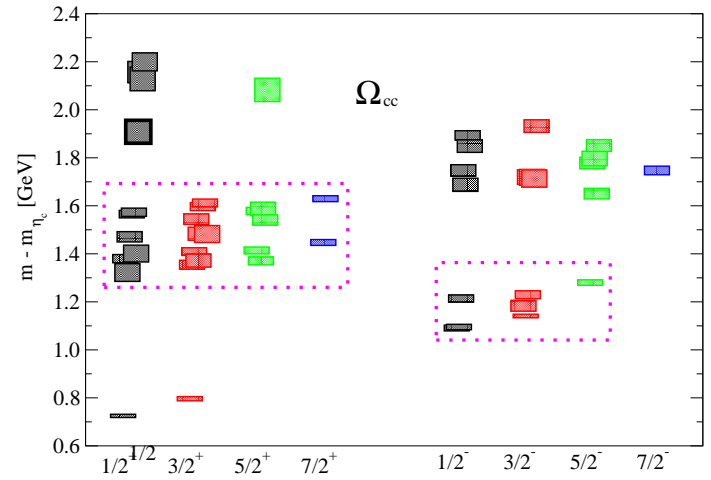

(a)

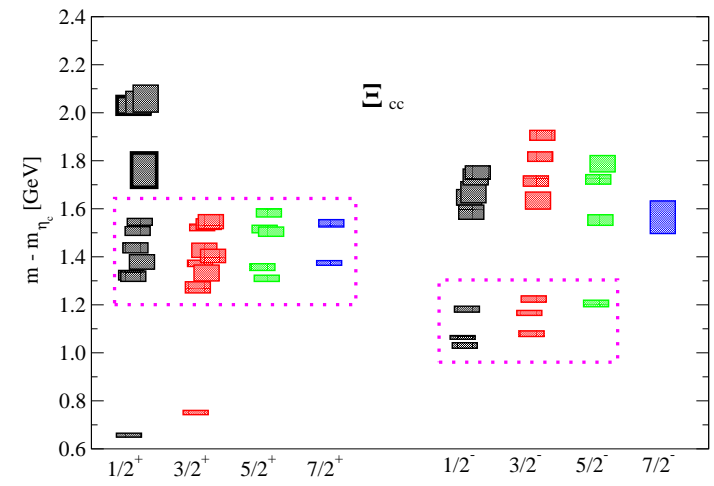

(b)

Figure 2: Spin identified spectra of (a) $\Omega_{c c}$ and (b) $\Xi_{c c}$ baryon for both parities and with spins up to $\frac{7}{2}$. The keys are same as in Figure 1(a). 
Figure 2 shows the spin identified spectra of the doubly charmed baryons. Here the spectra is shown with the mass of $\eta_{c}$ subtracted from them so as to account for the difference in the charm quark content. The boxes and the pink ellipses represent the similar quantities as in Figure 1. Here again one can see the agreement between the number of states in the lower non-relativistic bands and the expectations as shown in the Table 2(b) providing a clear signature of $\mathrm{SU}(6) \otimes \mathrm{O}(3)$ symmetry in the doubly charm baryon spectra also.

The spin dependent energy splittings provide important information about the nature of the interactions between different excitations. The most notable baryon energy splitting are the splittings due to spin-orbit coupling and the hyperfine splittings between spin- $\frac{3}{2}^{+}$and $\frac{1}{2}^{+}$states. In Figure 3 we plot the absolute values of energy differences between energy levels which originate from the spin-orbit interaction of the following (L, S) pairs : (2,3/2-in the left), (2,1/2-in the middle) and (1,1/2-in the right column). We plot these splittings at varying quark masses from light to bottom. We identified these $(\mathrm{L}, \mathrm{S})$ pairs by finding the operators which incorporate these pairs and which have major overlaps to these states. For bottom baryons we used the data from Ref. [18], and for light and strange baryon re-

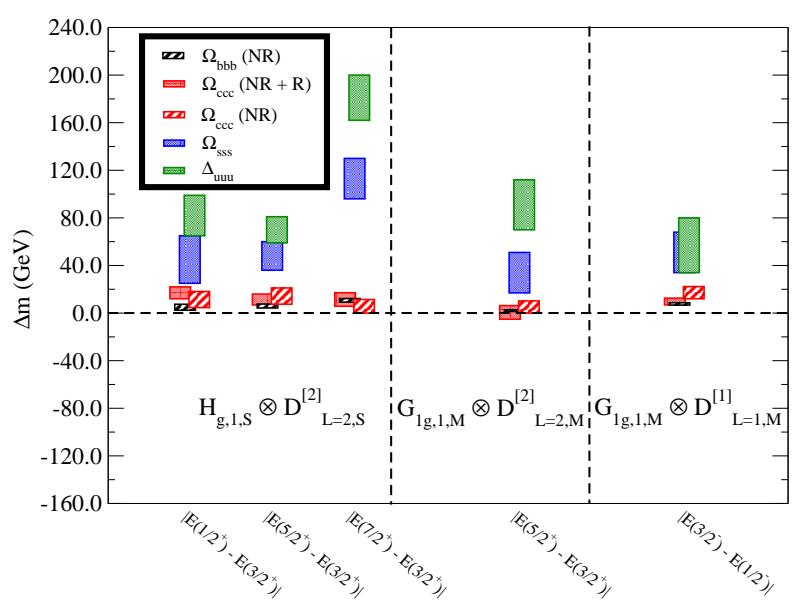

Figure 3: Energy splittings between states with same L and $S$ values, starting from light to heavy triple-flavoured baryons. For $\Omega_{b b b}$, results are with only non-relativistic operators [18]. For $\Omega_{c c c}$, results from relativistic and nonrelativistic as well as only non-relativistic operators are shown, and for the light and strange baryons results are with relativistic and non-relativistic operators [13]. sults from Ref. [13] are used. As one can see the degeneracy between these states is more or less satisfied for charm baryons [10] as is also observed in bottom baryons. However, data with higher statistics is necessary to identify conclusively the breaking of this degeneracy at charm quark.

For doubly-charmed baryons, in Figure 4, we plot the hyperfine splittings between spin- 3/2 and $1 / 2$ along with other lattice estimations as well as with various other potential model calculations [19-24]. It is to be noted that our results for $\Xi_{c c}$ are at pion mass $396 \mathrm{MeV}$. However results from Ref. [5, 6, 8, 9] are extrapolated to the physical pion mass, while the Ref. [4] results are at pion mass $390 \mathrm{MeV}$ and $340 \mathrm{MeV}$ (black circle).

In the heavy quark limit, naively one can expand the mass of a heavy hadron, with $n$ heavy quarks as $m_{H_{n q}}=n m_{Q}+A+B / m_{Q}+\mathscr{O}\left(1 / m_{Q^{2}}\right)$ [25]. Hence the energy splittings between the heavy hadrons can be expressed in the form $a+b / m_{Q}$. In Figure 5 we plot the energy splittings of spin- $\frac{1}{2}$ and $\frac{3}{2}$ triple-flavored baryons from the isoscalar vector meson ground states against the square of the pseudoscalar masses. Data for the light and strange are taken from Ref. [13], while the numbers for bottom quark are taken from Ref. [18] which uses a non-relativistic action. The shaded regions are the extrapolations based on results from fitting the data to the above form, excluding the light sector. One can observe that for various fits the fit estimates even pass through 

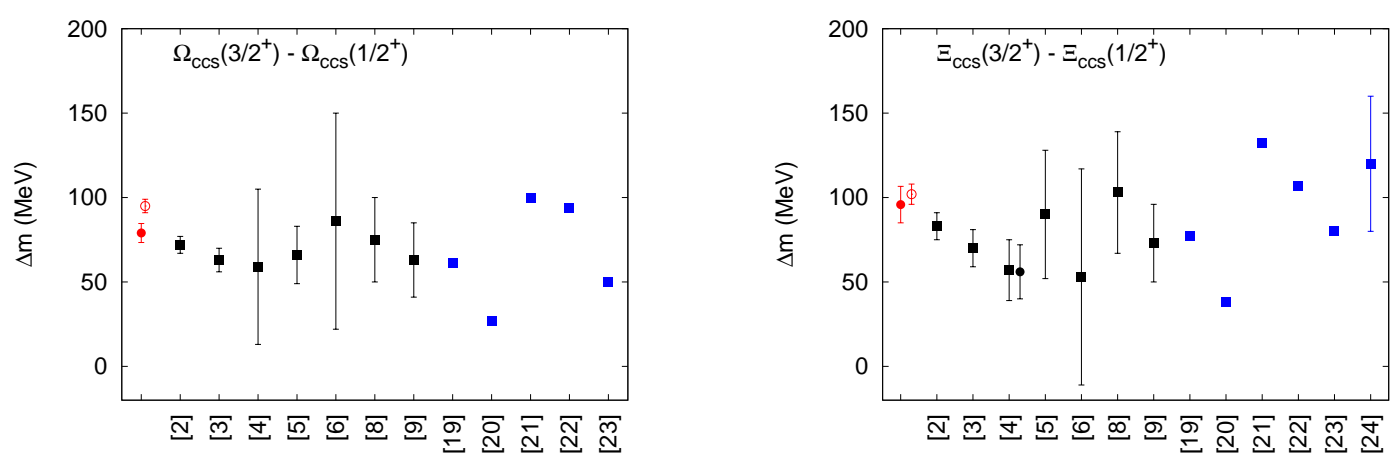

Figure 4: Hyperfine splittings of $\Omega_{c c}$ and $\Xi_{c c}$ baryons are compared for various lattice and potential model calculations. The red circles are estimates from this work. The filled red circle is from $c_{s w}=1.35$, while the unfilled red circle is estimated from a boosted $c_{s w}=2.0$. The black squares represents other lattice results. The blue squares are estimates from various potential model calculations.

the light data even though they are not included in the fitting. We also make similar observations in other spin-parity channels. Study of such energy splittings in the doubly charm sector allows us to make predictions in the bottom hadrons. Fitting $\left(m_{\Xi_{c c}^{*}}-m_{D}, m_{\Omega_{c c}^{*}}-m_{D_{s}}, m_{\Omega_{c c c}}-m_{\eta_{c}}\right)$ we get a prediction for $m_{\Omega_{c c c}}=8050 \pm 10 \mathrm{MeV}$, and fitting of $\left(m_{\Xi_{c c}^{*}}-m_{D^{*}}, m_{\Omega_{c c}^{*}}-m_{D_{s}^{*}}, m_{\Omega_{c c c}}-m_{J / \psi}\right)$ allow us to predict $m_{B_{c}^{*}}-m_{B_{c}}=80 \pm 8 \mathrm{MeV}$.

\section{Conclusions}

In this work we present the first calculation of the ground and excited state spectra of doubly and triply-charmed baryons by using dynamical lattice QCD. The spectra that we obtain have states with well-defined total spins up to $7 / 2$ and the low lying states remarkably resemble the expectations of quantum numbers from $\mathrm{SU}(6) \otimes \mathrm{O}(3)$ symmetry. Various energy splittings including splittings due to hyperfine as well as spin-orbit coupling were studied and those are compared against the same energy splittings at other quark masses. From these energy splitting studies we also make predictions in the bottom sector which are $m_{B_{c}^{*}}-m_{B_{c}}=80 \pm 8 \mathrm{MeV}$ and $m_{\Omega_{c c b}}=8050 \pm 10 \mathrm{MeV}$. However, it is to be noted that we only mentioned statistical error in this work and the systematics from other sources like chiral extrapolation, lattice spacing are not addressed here. Also we have not incorporated multi-hadron operators which may effect some of the above conclusions, though to a lesser extent than their influence in the light hadron spectra.
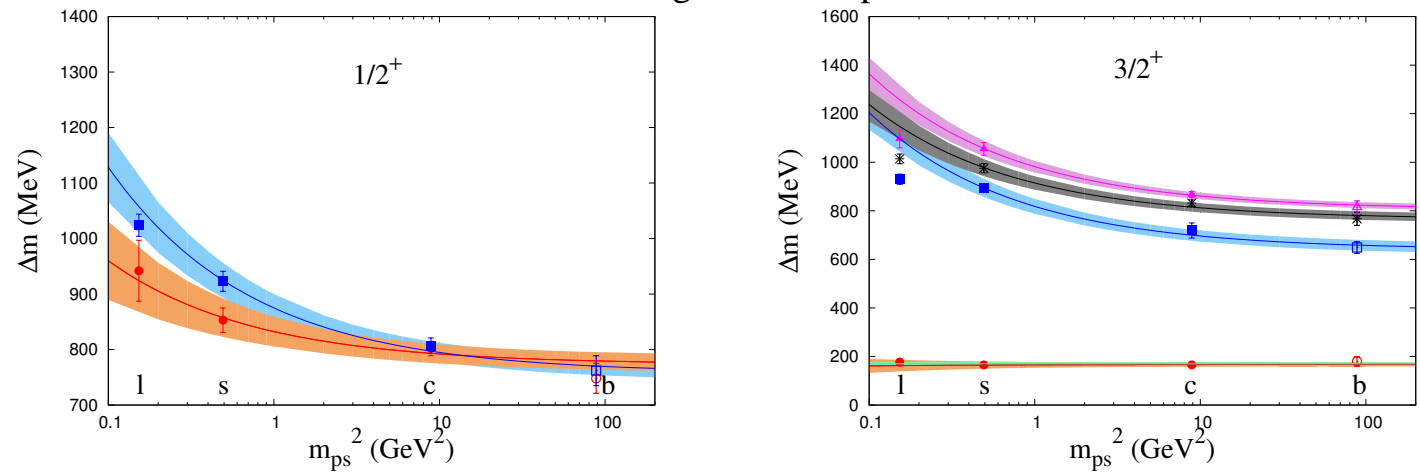

Figure 5: Energy splittings of spin- $\frac{1}{2}$ and $\frac{3}{2}$ triple-flavored baryons from the isoscalar vector meson ground states are plotted against the square of the pseudo scalar meson masses. The keys for light, strange and bottom data are the same as in Figure 2. Shaded regions are the extrapolations based on results from fitting the strange, charm and bottom data to a heavy quark inspired form $a+b / m_{Q}$. 


\section{Acknowledgements}

We thank our colleagues within the Hadron Spectrum Collaboration. Chroma [26] and QUDA [27, 28] were used to perform this work on the Gaggle and Brood clusters of the Department of Theoretical Physics, Tata Institute of Fundamental Research and at Lonsdale cluster maintained by the Trinity Centre for High Performance Computing and at Jefferson Laboratory. MP acknowledges support from the Trinity College Dublin Indian Research Collaboration Initiative and the CSIR, India for financial support through the SPMF.

\section{References}

[1] The Review of Particle Physics : J. Beringer et al. (PDG), Phys. Rev. D86, 010001 (2012).

[2] R. Lewis, N. Mathur and R. M. Woloshyn, Phys. Rev. D 64, 094509 (2001).

[3] N. Mathur, R. Lewis and R. M. Woloshyn, Phys. Rev. D 66, 014502 (2002).

[4] S. Basak, S. Datta, M. Padmanath, P. Majumdar and N. Mathur, PoS LATTICE 2012, 141 (2012); PoS LATTICE 2013, paper in preparation.

[5] C. Alexandrou et al., Phys. Rev. D 86, 114501 (2012).

[6] R. A. Briceno, H. -W. Lin and D. R. Bolton, Phys. Rev. D 86, 094504 (2012).

[7] S. Durr, G. Koutsou and T. Lippert, Phys. Rev. D 86, 114514 (2012) [arXiv:1208.6270 [hep-lat]].

[8] Y. Namekawa et al. [PACS-CS Collaboration], arXiv:1301.4743 [hep-lat].

[9] G. Bali, S. Collins and P. Perez-Rubio, J. Phys. Conf. Ser. 426, 012017 (2013).

[10] M. Padmanath, R. G. Edwards, N. Mathur and M. Peardon, arXiv:1307.7022 [hep-lat].

[11] R. G. Edwards, B. Joo and H. -W. Lin, Phys. Rev. D 78, 054501 (2008) [arXiv:0803.3960 [hep-lat]].

[12] H. -W. Lin et al. [Hadron Spectrum Collaboration], Phys. Rev. D 79, 034502 (2009)

[13] R. G. Edwards, J. J. Dudek, D. G. Richards and S. J. Wallace, Phys. Rev. D 84, 074508 (2011).

[14] M. Peardon et al. [Hadron Spectrum Collaboration], Phys. Rev. D 80, 054506 (2009)

[15] J. J. Dudek, R. G. Edwards, N. Mathur and D. G. Richards, Phys. Rev. D 77, 034501 (2008).

[16] J. J. Dudek and R. G. Edwards, Phys. Rev. D 85, 054016 (2012).

[17] L. Liu et al. [Hadron Spectrum Collaboration], JHEP 1207, 126 (2012) [arXiv:1204.5425 [hep-ph]].

[18] S. Meinel, Phys. Rev. D 85, 114510 (2012).

[19] W. Roberts and M. Pervin, Int. J. Mod. Phys. A 23, 2817 (2008).

[20] A. P. Martynenko, Phys. Lett. B 663, 317 (2008).

[21] V. V. Kiselev et al., Phys. Usp. 45, 455 (2002);

[22] D. Ebert et al., Phys. Rev. D 66, 014008 (2002).

[23] J. G. Korner, M. Kramer and D. Pirjol, Prog. Part. Nucl. Phys. 33, 787 (1994).

[24] N. Brambilla, A. Vairo and T. Rosch, Phys. Rev. D 72, 034021 (2005).

[25] E. E. Jenkins, Phys. Rev. D 54, 4515 (1996).

[26] R. G. Edwards et al., Nucl. Phys. Proc. Suppl. 140, 832 (2005) [hep-lat/0409003].

[27] M. A. Clark et al, Comput. Phys. Commun. 181, 1517 (2010).

[28] R. Babich, M. A. Clark and B. Joo, arXiv:1011.0024 [hep-lat]. 